Boise State University

ScholarWorks

$12-2004$

\title{
Effects of Signal Processing and Antenna Frequency on the Geostatistical Structure of Ground-Penetrating Radar Data
}

Greg A. Oldenborger

Boise State University

Michael D. Knoll

Boise State University

Warren Barrash

Boise State University 


\title{
Effects of Signal Processing and Antenna Frequency on the Geostatistical Structure of Ground-Penetrating Radar Data
}

\author{
Greg A. Oldenborger ${ }^{1}$, Michael D. Knoll and Warren Barrash \\ Center for Geophysical Investigation of the Shallow Subsurface, \\ Boise State University, Boise, Idaho 83725
}

\begin{abstract}
Recent research has suggested that the geostatistical structure of ground-penetrating radar data may be representative of the spatial structure of hydraulic properties. However, radar images of the subsurface can change drastically with application of signal processing or by changing the signal frequency. We perform geostatistical analyses of surface radar reflection profiles in order to investigate the effects of data processing and antenna frequency on the semivariogram structure of radar reflection amplitudes. Surface radar reflection data collected at the Boise Hydrogeophysical Research Site illustrate the processing- and antenna-dependence of radar semivariograms for a fluvial, cobble-andsand aquifer. Compensating for signal attenuation and spreading using a gain function removes a nonstationary trend from the data and a trace-specific gain function reduces fluctuation of semivariogram values at large lags. Otherwise, geostatistical structures of surface reflection data are quite robust to the effects of data gains. Migration is observed to reduce the strength of diffraction features in the semivariogram fields and to increase the principal exponential range. Principal exponential range increases only slightly after application of migration with a realistic velocity but over-migration results in a significant artificial increase of exponential range. The geostatistical structures of radar reflection data exhibit marked dependence on antenna frequency, thus highlighting the critical importance of the scale of measurement. Specifically, the exponential ranges of radar reflection amplitudes decrease in proportion to the increased signal frequency for the $50 \mathrm{MHz}, 100 \mathrm{MHz}$ and $200 \mathrm{MHz}$ range of antennas. Results demonstrate that processing and antenna frequency must be considered before the application of radar reflection data in a geostatistical context.
\end{abstract}

\section{Introduction}

In a hydrogeophysical context, ground-penetrating radar (GPR) surveys find application in contaminant characterization studies, subsurface structure mapping and hydraulic property estimation (Knight, 2001). With respect to the latter application, Olhoeft (1994) investigated the spatial persistence of radar reflections and Knight et al. (1996) and Rea and Knight (1998) advanced the hypothesis that the geostatistical structure of radar reflection amplitudes, as quantified by the semivariogram, could be used to infer the geostatistical structure of hydraulic properties. This hypothesis was based on the facts that radar reflections in the subsurface are caused by changes in the dielectric properties of the geologic materials and that the petrophysical parameters that determine the dielectric properties of a sedimentary unit (grain size, composition and packing) also determine the hydraulic properties.

While a direct relationship between the geostatistical structure of radar reflection amplitudes and geologic or hydraulic properties has yet to be demonstrated (Oldenborger

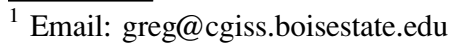

et al., 2003), geostatistical analysis of radar reflection data has been applied in the extrapolation of hydrostratigraphy (Langsholt et al., 1998), in depositional environment comparisons (Tercier et al., 2000), and in reservoir characterization where the geostatistical structure of radar reflection data was used to interpolate and extrapolate the permeability field within an unsaturated sandstone formation (Szerbiak et al., 2001). Other workers may be considering or applying similar techniques as a method of obtaining critical information regarding the geologic or hydraulic property distribution away from control points. Given an increasing utilization of the technique, it is important to realize that the reliable geostatistical use of radar data may be further complicated by the dependence of the reflection image on data processing and antenna frequency.

The Boise Hydrogeophysical Research Site (BHRS) is a specific example of a site where geostatistical analysis of radar reflection data might allow interpolation and extrapolation of hydraulic properties away from borehole measurements. The BHRS is a meso-scale research site consisting of 18 boreholes completed in a shallow, fluvial cobble and sand aquifer located alongside the Boise River near Boise, Idaho. Currently, borehole-based measurements are 
insufficient for determining the horizontal geostatistical structure of porosity within the sedimentary units (Barrash and Clemo, 2002). Surface GPR surveys comprise part of the geophysical characterization at the BHRS; reflection data have been interpreted in terms of sedimentary units and bounding surfaces (Peretti et al., 1999) and have proven useful for identification of sedimentary architecture between and away from boreholes. Before the radar data can be further used as a proxy for the geostatistical structure of porosity, we must quantify the effects of signal processing and antenna frequency on the geostatistical structure of the radar reflection amplitudes.

In particular, three questions are posed: 1) How is the geostatistical structure of surface GPR reflection data effected by different data gains? 2) How is the geostatistical structure of GPR data effected by migration? 3) How does the geostatistical structure of GPR data vary with signal frequency? We address these questions by analyzing the geostatistics of surface radar reflection data collected at the BHRS. Observed variation of geostatistical model parameters (such as exponential range) is used to quantify the sensitivity of geostatistics to changes in the processing scheme or the radar antenna frequency.

\section{Data Acquisition and Processing}

Surface radar reflection data were collected at the BHRS in the summer of 1998 (Peretti et al., 1999). These data were collected with a PulseEKKO 100 (Sensors and Software Inc.) bistatic radar system with $50 \mathrm{MHz}$ antennas and a 1,000 $\mathrm{V}$ transmitter, with $100 \mathrm{MHz}$ antennas and a $400 \mathrm{~V}$ transmitter, and with $200 \mathrm{MHz}$ antennas and a $400 \mathrm{~V}$ transmitter. Survey design and acquisition parameters followed established protocol for constant-offset GPR (Annan, 1999). Horizontal and temporal sampling intervals were $0.1 \mathrm{~m}$ and $0.8 \mathrm{~ns}$ for the $200 \mathrm{MHz}$ and $100 \mathrm{MHz}$ data, and $0.2 \mathrm{~m}$ and $1.6 \mathrm{~ns}$ for the $50 \mathrm{MHz}$ data.

Traditional seismic processing methods can be applied to ground-penetrating radar data (for example, Fisher et al., 1992; Greaves et al., 1996) provided that the user accounts for the inherent differences in sources and expressions of noise, and energy loss mechanisms. These specifications are especially important when attempting to retain relative radar reflection amplitude information due to the fundamental differences in the dynamics of seismic and electromagnetic wave propagation.

With these caveats in mind, the processing flow for this study is: 1) trace editing to ensure correct mid-point location and addition of elevation information to trace headers; 2) removal of zero-frequency bias as calculated based on samples up to the first break; 3) horizontal alignment (in time) of first breaks; 4) removal of signal saturation noise via a residual median filter designed according to Gerlitz et al. (1993); 5) 3-point median filtering of occasional spurious noise spikes; 6) topographic corrections using a 0.2 ns sample interval and a velocity equal to that of the unsaturated zone as determined from cross-borehole transmission and vertical radar profile data; 7) application of data gain; 8) constantvelocity phase-shift migration for data below the water table (Gazdag, 1978); 9) muting of the highly coherent air/ground waves and the water table reflection.

Application of an appropriate data gain requires some discussion. Gains such as automatic gain control (AGC) that attempt to equalize signal strength are considered to be inappropriate due to their destruction of relative amplitude information (Yilmaz, 2001). Conversely, programmed gain control (PGC; Yilmaz, 2001) relies on the assertion that the amplitude fall-off envelope is a reliable attribute that describes amplitude decay. Accordingly, the gain function is the inverse of the fall-off envelope. To preserve relative amplitude variations in the lateral direction, a single (average) PGC function is often applied to an entire reflection profile. However, for profiles with lateral variation in material properties (and, therefore, attenuation) or lateral variations in antenna coupling, it may be necessary to apply PGC gain on a trace-by-trace basis. Similarly, model-based gains such as spreading exponential compensation (SEC; Annan, 1999) may be applicable for maintaining relative amplitude information on either a whole-section or trace-bytrace basis provided that appropriate estimates of physical properties are used. In subsequent geostatistical analysis, we examine observable variations in the radar geostatistics due to a selection of different data gains.

\section{Geostatistical Estimators}

Definition of geostatistical estimators is covered extensively by Isaaks and Srivastava (1989) and Deutsch and Journel (1998). The normalized semivariogram for a random variable $g(\mathbf{r})$ can be defined in terms of the expected squared difference between variable values separated by a lag vector $\mathbf{h}$ :

$$
\gamma(\mathbf{h})=\frac{1}{2 \sigma^{2}} E\left\{[g(\mathbf{r})-g(\mathbf{r}-\mathbf{h})]^{2}\right\}
$$

where $\mathbf{r}$ is the position vector, $E$ is the expectation operator and $\sigma^{2}$ is the variance. The normalized semivariogram (hereafter referred to as the semivariogram) can be understood as the sample variance described as a function of spatial separation. Thus, low semivariogram values indicate a high degree of correlation between variable values separated by the lag vector. For this and other studies (Rea and Knight, 1998; Tercier et al., 2000; Szerbiak et al., 2001; Oldenborger et al., 2003), the variable $g$ of Equation (1) is radar reflection amplitude.

Often, the semivariogram is calculated using a directional, pair-wise comparison algorithm (Deutsch and Journel, 
1998). While such an algorithm is necessary for irregularly sampled data, regularly sampled data (such as GPR data) allow for spectral calculation of two-dimensional (2-D) semivariogram fields at a much lower computational cost. Spectral methods are employed in this study; details of frequencydomain transforms are provided by Bracewell (1986). Briefly, the spectral method of semivariogram calculation produces the 2-D autocorrelation (autocovariance) field

$$
r\left(h_{x}, h_{z}\right)=\operatorname{IFT} 2 \mathrm{D}\left\{G\left(k_{x}, k_{z}\right) G^{*}\left(k_{x}, k_{z}\right)\right\}
$$

where $G\left(k_{x}, k_{z}\right)$ is the 2-D Fourier transform of the variable $g(x, z), G^{*}$ is the complex conjugate of $G, k_{x}$ and $k_{z}$ are the horizontal and vertical wavenumbers respectively, and IFT2D \{\} represents the inverse 2-D Fourier transform. Normalization of the real part of the autocorrelation by the variance yields the 2-D correlogram field, and subtraction of the correlogram from unity yields the normalized 2-D semivariogram field. The 2-D semivariogram field can be thought of as a composite surface resulting from the interference of multiple spatial correlation functions and since the correlogram may fall below zero, the semivariogram may rise above unity. The topography of the 2-D semivariogram surface is easily examined by slicing in any direction to yield a onedimensional (1-D) semivariogram and attempts can be made to isolate the 1-D correlation trends.

Experimental semivariograms of radar data exhibit both transitional and periodic features (Rea and Knight, 1998; Oldenborger et al., 2003). In this study, transitional features are described using an exponential model as is commonly done for fluvial sedimentary environments (Woodbury and Sudicky, 1991), whereas the periodic features require application of a dampened hole effect model (Deutsch and Journel, 1998). Linear combination of the aforementioned models with a linear drift and nugget effect results in a general composite model of the form

$$
\begin{aligned}
\gamma(h)= & n+w\left[1-\exp \left(-\frac{3 h}{a}\right)\right] \\
& +w_{\mathrm{H}}\left[1-\exp \left(-\frac{3 h}{d_{\mathrm{H}}}\right) \cos \left(\frac{2 \pi h}{\lambda_{\mathrm{H}}}\right)\right]+m h
\end{aligned}
$$

where $h$ is the lag vector magnitude in a specified direction, $n$ is the nugget, $w$ is the exponential weight, $a$ is the exponential range, $w_{\mathrm{H}}$ is the hole weight, $\lambda_{\mathrm{H}}$ is the hole wavelength (i.e., twice the length of the periodic feature), $d_{\mathrm{H}}$ is the damping distance and $m$ is the linear drift. Deutsch and Journel (1998) further discuss properties and parameters of the various models and their linear combination or nesting. For $m=0$, the semivariogram model will plateau at some sill equal to the sum of the nugget and the weights. For normalized semivariograms of this type, the sill will be close to unity. Weights describe the portion of the sample variance attributed to each component of the nested model. Normalizing the semivariogram allows for comparison of relative weights, nuggets and

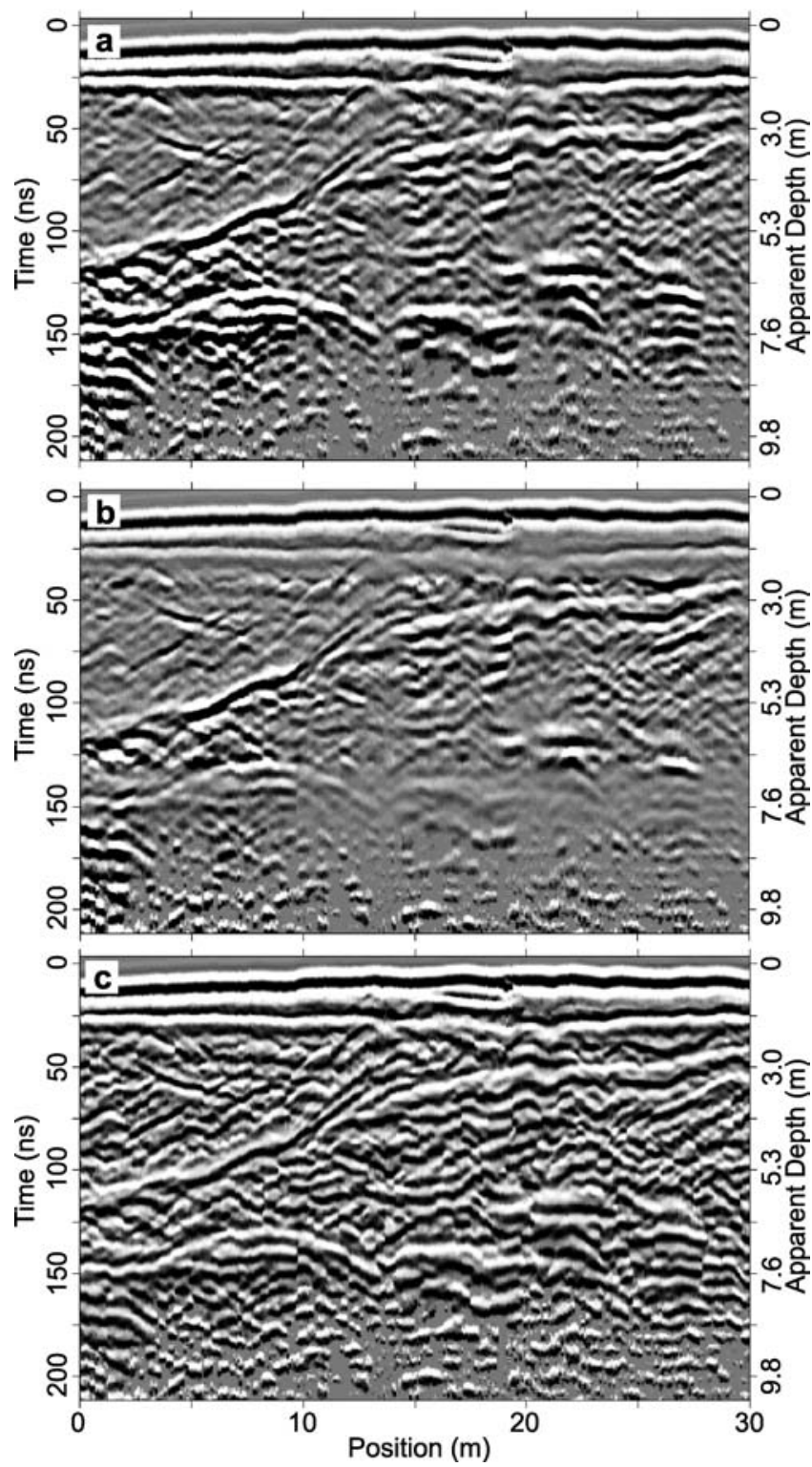

Figure 1. $200 \mathrm{MHz}$, small-offset, BHRS reflection profile with application of (a) SEC, (b) PGC, and (c) trace-by-trace PGC gain functions. Apparent depth conversion velocity is $0.091 \mathrm{~m} / \mathrm{ns}$.

linear drift for different data sets that may have different sample variances such as gained and non-gained radar data.

Model parameters are fit to the experimental geostatistics based on minimization of the sum of the squares of the residuals between the experimental and model semivariograms for lags greater than zero and less than one-half the maximum extent of the data.

\section{Geostatistical Analysis of Radar Data}

To isolate the effects of data processing and antenna frequency on geostatistical structure, we discuss the 
Journal of Environmental and Engineering Geophysics
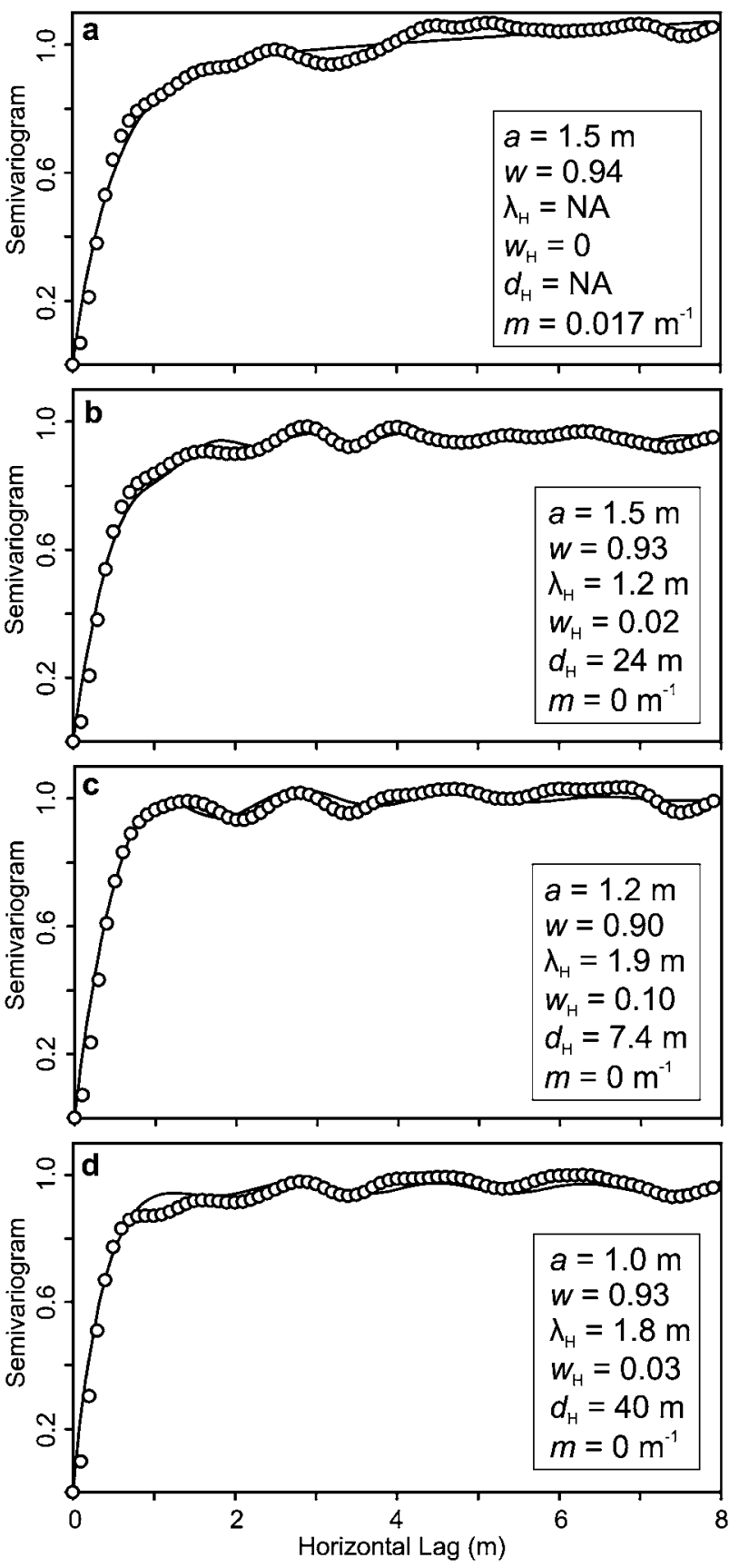

Figure 2. Horizontal semivariograms for $200 \mathrm{MHz}$ reflection amplitudes over the 45-160 ns time window with application of the following gain functions: (a) none, (b) SEC, (c) PGC, and (d) trace-by-trace PGC. Points indicate experimental geostatistics; solid lines indicate least-squares models corresponding to the listed model parameters. All models have zero nugget.

geostatistics for a single $30 \mathrm{~m}$ reflection line running through the center of the BHRS. The reflection line is approximately perpendicular to the paleo-flow direction of the fluvial deposits. While hierarchical behavior has been

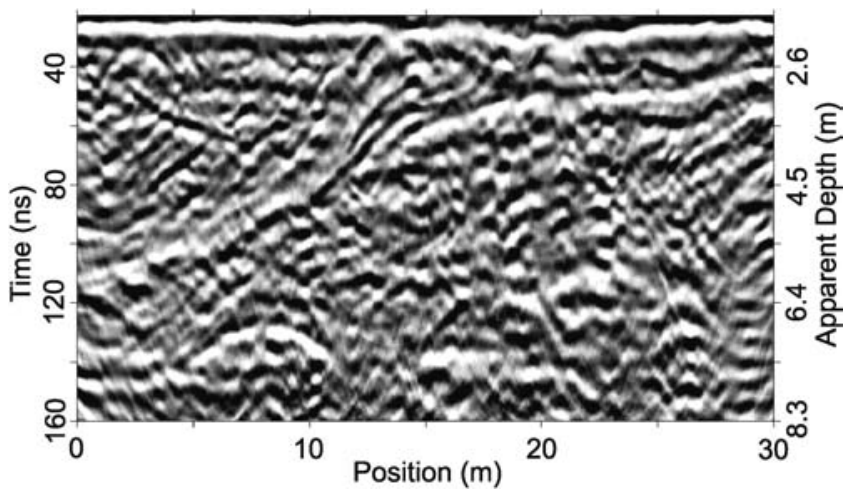

Figure 3. Migrated $200 \mathrm{MHz}$ reflection data. Migration velocity is $0.095 \mathrm{~m} / \mathrm{ns}$. The time axis is the original sample time and is displayed to indicate the geostatistical window of 45-160 ns.

identified for the porosity field of these fluvial deposits (Barrash and Clemo, 2002), available ground truth is insufficient for conclusive hierarchical geostatistical characterization of the radar reflection data. Instead, we focus on changes of the radar geostatistics for a constant portion of the reflection image as processing parameters and antenna frequency are varied. If two separate data sets contain equivalent spatial information, the equivalency should be observed in the geostatistical model parameters regardless of direction or hierarchical structures. Vertical geostatistics of reflection data are dominated by the strong periodicity of each reflection that is due to the wavelet nature of the radar pulse (Rea and Knight, 1998) and are not discussed herein.

\section{Gain Variation}

The $200 \mathrm{MHz}$ BHRS reflection profile is shown in Fig. 1 with the application of three different gain functions: SEC, PGC, and trace-by-trace PGC. To accommodate the 2-D nature of the geostatistical estimator, two-way traveltime must be converted to depth. A depth conversion velocity of $0.091 \mathrm{~m} / \mathrm{ns}$ is obtained from cross-borehole level-run data from the water table to $8 \mathrm{~m}$ depth $(160 \mathrm{~ns})$ at which point the $200 \mathrm{MHz}$ reflection data become too attenuated for analysis. Geostatistical analysis (for these and all subsequent data) is performed on data within the time window of 45-160 ns as defined by the water table reflection and the $200 \mathrm{MHz}$ attenuation limit. For migrated data, this time window ensures that the same physical reflectors will be included in all analyses although the migrated depths to the reflectors may be different.

Horizontal semivariograms of the $200 \mathrm{MHz}$ gained reflection data are displayed in Fig. 2. Geostatistical parameters of the differently-gained $200 \mathrm{MHz}$ profiles are remarkably similar given the large visual differences apparent in Fig. 1. This is because even though the gains are non-linear and variable, there is no spatial redistribution of amplitude 
Oldenborger et al.: Geostastistical Structure of Ground-Penetrating Radar Data
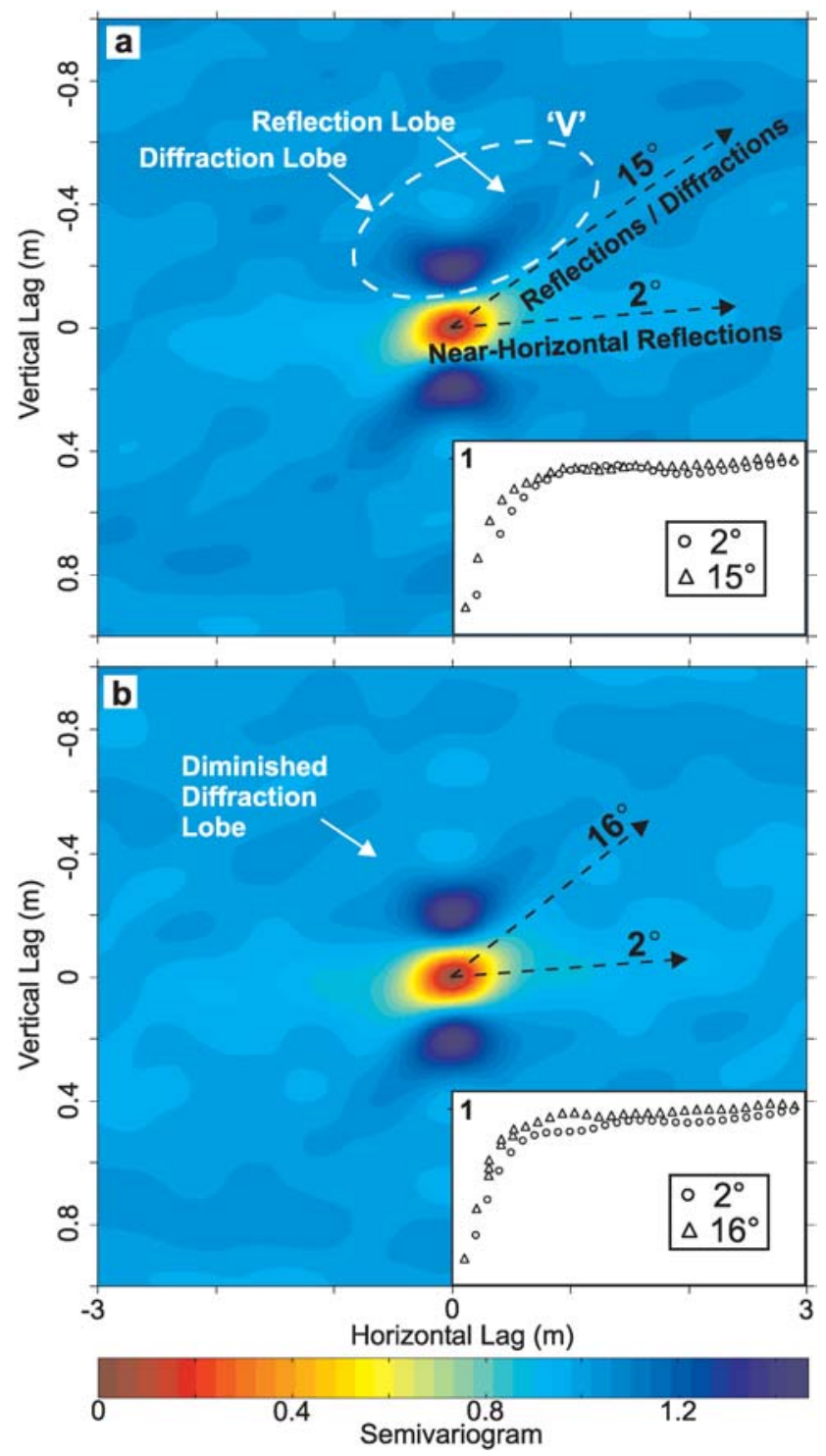

Figure 4. Semivariogram fields for (a) depth-converted and (b) migrated $200 \mathrm{MHz}$ reflection amplitudes of Figs. $1 c$ and 3 respectively. The inset in (a) shows the surface topography at $2^{\circ}$ (circles) and $15^{\circ}$ (triangles) for which the exponential ranges are $1.1 \mathrm{~m}$ and $0.9 \mathrm{~m}$ respectively. The inset in (b) shows the surface topography at $2^{\circ}$ (circles) and $16^{\circ}$ (triangles) for which the exponential ranges are $1.2 \mathrm{~m}$ and $0.8 \mathrm{~m}$ respectively. Depth is defined as positive downwards such that a negative vertical lag is upwards. Note that the horizontal-to-vertical aspect ratio is greater than unity such that angles are compressed in the vertical direction.

information; local maxima remain local maxima and zero crossings remain zero crossings. Furthermore, because gains are primarily vertically-varying functions, pre- and post-gain squared differences in amplitude (normalized to the pre- and post-gain variance) will be similar in the horizontal direction.
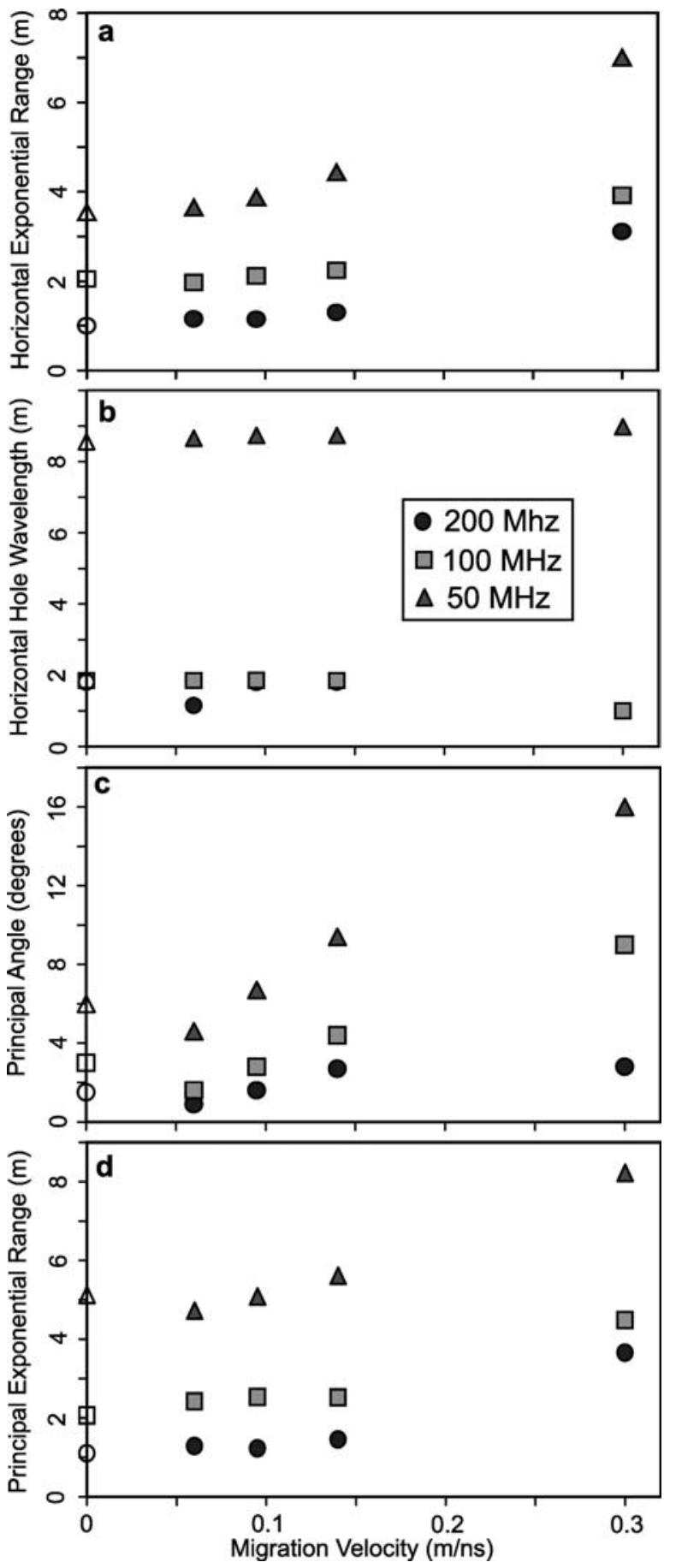

Figure 5. Effects of migration velocity on (a) horizontal exponential range, (b) horizontal wavelength, (c) principal angle, and (d) principal exponential range. Circle, square and triangle symbols indicate 200 , 100 and $50 \mathrm{MHz}$ data respectively. Open symbols at zero velocity indicate geostatistical parameters for the depth-converted rather than migrated data. 
Table 1. Effect of migration velocity on the geostatistical parameters for the $200 \mathrm{MHz}$ trace-by-trace PGC-gained reflection data set; $v$ denotes the migration velocity; $\phi$ denotes principal correlation angle; the subscript 0 denotes the horizontal direction. Geostatistical model parameters are defined in the text; $n=0$ for all models except $n_{\phi}=0.08$ for $v=0.3 \mathrm{~m} / \mathrm{ns}$. The increase of principal angle with migration velocity results from increased time stretching.

\begin{tabular}{lccccccccccc}
\hline \hline $\boldsymbol{v}(\mathbf{m} / \mathbf{n s})$ & $\boldsymbol{a}_{\mathbf{0}}(\mathbf{m})$ & $\boldsymbol{w}_{\mathbf{0}}$ & $\boldsymbol{\lambda}_{\mathbf{H} \mathbf{0}}(\mathbf{m})$ & $\boldsymbol{w}_{\mathbf{H} \mathbf{0}}$ & $\boldsymbol{d}_{\mathbf{H} \mathbf{0}}(\mathbf{m})$ & $\left.\boldsymbol{\phi} \boldsymbol{(}^{\circ}\right)$ & $\boldsymbol{a}_{\boldsymbol{\phi}}(\mathbf{m})$ & $\boldsymbol{w}_{\boldsymbol{\phi}}$ & $\boldsymbol{\lambda}_{\mathbf{H} \boldsymbol{\phi}}(\mathbf{m})$ & $\boldsymbol{w}_{\mathbf{H} \phi}$ & $\boldsymbol{d}_{\mathbf{H} \boldsymbol{}}(\mathbf{m})$ \\
\hline $0.091^{\mathrm{a}}$ & 1.0 & 0.93 & 1.8 & 0.02 & 40 & 2 & 1.1 & 0.93 & 2.2 & 0.04 & $\infty$ \\
0.06 & 1.2 & 0.93 & 1.2 & 0.05 & 5.8 & 1 & 1.3 & 0.95 & 1.2 & 0.04 & 12 \\
0.095 & 1.2 & 0.95 & 1.8 & 0.02 & 48 & 2 & 1.2 & 0.95 & 2.1 & 0.02 & $\infty$ \\
0.14 & 1.3 & 0.96 & 1.8 & 0.03 & 30 & 2 & 1.4 & 0.94 & 2.1 & 0.03 & 13 \\
0.3 & 3.1 & 0.99 & $\mathrm{NA}$ & 0 & $\mathrm{NA}$ & 3 & 3.7 & 0.95 & $\mathrm{NA}$ & 0 & NA \\
\hline
\end{tabular}

${ }^{a}$ Depth conversion only.

All of the gained profiles are best modeled with nested exponential and hole effect models with similar ranges and wavelengths (Fig. 2). Hole effects occur as small (less than $10 \%)$ periodic oscillations about the exponential functions and are attributable to dipping reflectors that are cross-cut by the horizontal lag vector. The horizontal hole wavelengths are nearly twice the expected wavelength of the vertical radar
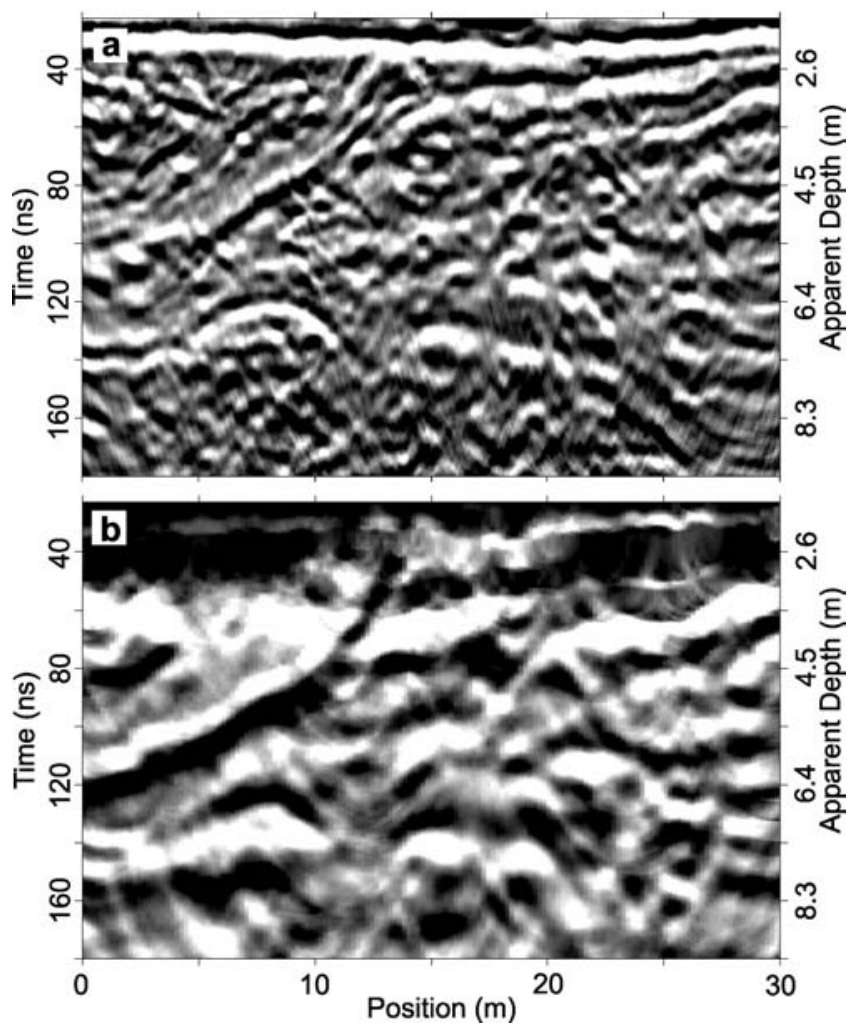

Figure 6. Migrated 100 and $50 \mathrm{MHz}$ reflection data. Migration velocity is $0.095 \mathrm{~m} / \mathrm{ns}$. The time axis is the original sample time and is displayed to indicate the geostatistical window of 45-160 ns. signal in the saturated zone; this observation is consistent with reflector dips of $10-20^{\circ}$. The depth-converted reflection profiles suggest geological dips up to approximately $13^{\circ}$ and diffraction tail dips up to approximately $17^{\circ}$ (Fig. 1).

Despite the similarity of model semivariograms, there are effects of gain on the experimental geostatistics that are manifest in the semivariogram models. It is observed that the non-gained data require a small linear drift component of the semivariogram model which suggests that the reflection amplitudes are non-stationary (Fig. 2a). All of the gain functions are effective at removing the linear drift. Furthermore, at lags approaching half of the maximum separation (not shown), the SEC- and PGC-gained data exhibit more erratic semivariograms when compared to that of the traceby-trace PGC-gained data. This is a result of application of a single gain function to an entire profile over which material properties and antenna coupling are variable. Trace-by-trace PGC gain (used exclusively hereafter) accounts for horizontal variations of attenuation and coupling and, therefore, results in a more stationary reflection image (Fig. 1c) and a more stable semivariogram at large lags.

\section{Migration Effects}

Practically, the process of migration acts to collapse diffraction features and rotate dipping reflectors into true subsurface locations. Thus, contrary to simple gain functions and time-depth conversion, migration involves a redistribution of reflected amplitudes within the image plane and potential rotation of the principal correlation directions (defined as the direction of maximum range). For this reason, it is informative to compare entire 2-D semivariogram fields. Figure 3 illustrates the result of application of constant-velocity Gazdag migration to the $200 \mathrm{MHz}$ traceby-trace PGC-gained reflection data below the water table. Migration velocity analysis results in an estimated velocity of $0.095 \mathrm{~m} / \mathrm{ns}$ which is very similar to the average crossborehole transmission velocity of $0.091 \mathrm{~m} / \mathrm{ns}$. 
Oldenborger et al.: Geostastistical Structure of Ground-Penetrating Radar Data
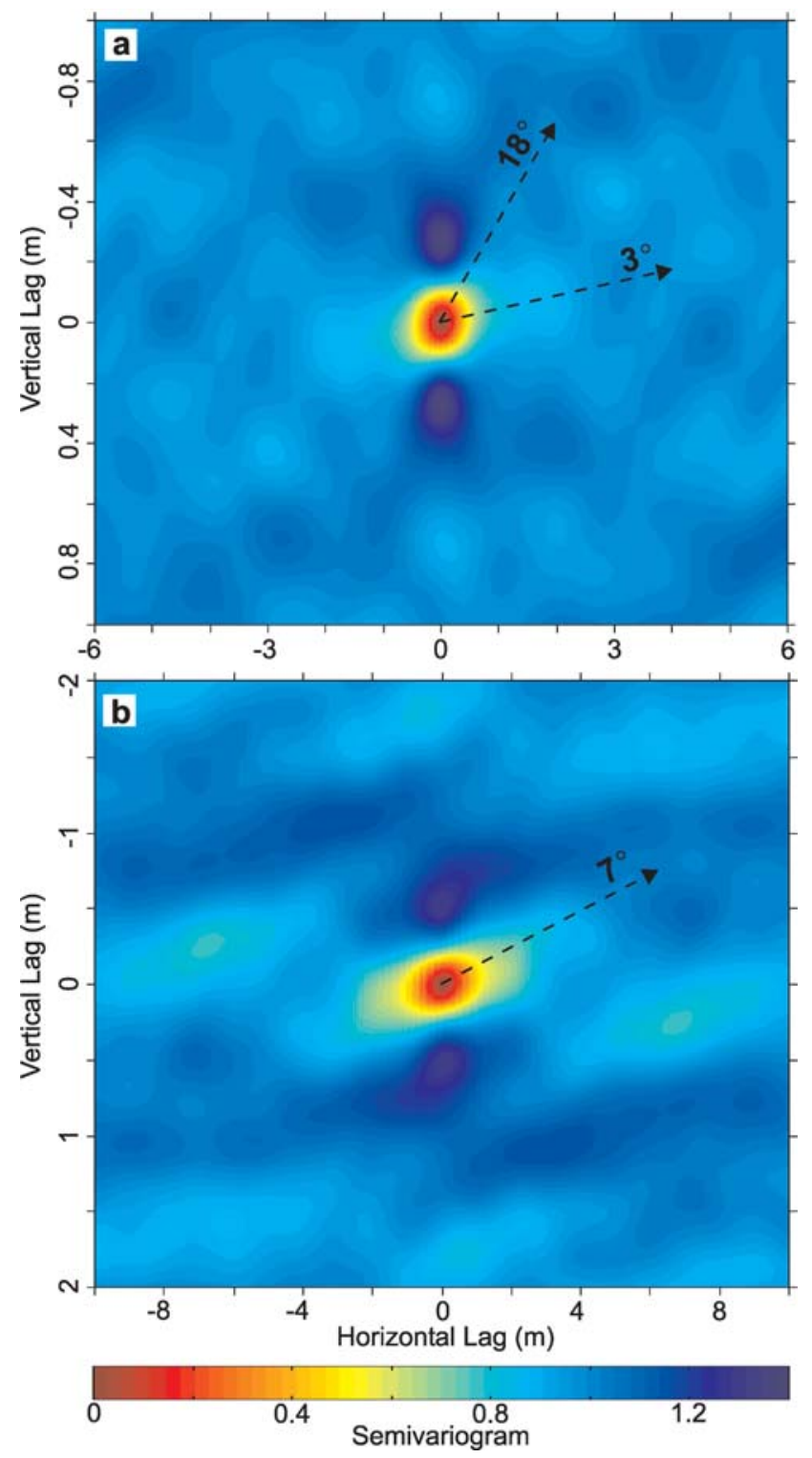

Figure 7. Semivariogram fields for migrated (a) 100 MHz and (b) $50 \mathrm{MHz}$ reflection amplitudes of Fig. 6. Depth is defined as positive downwards such that a negative vertical lag is upwards. Note that the horizontal-to-vertical aspect ratio is greater than unity such that angles are compressed in the vertical direction.

Figure 4 shows the semivariogram fields for the depthconverted and migrated $200 \mathrm{MHz}$ data. Figure 2d represents the slice of the 2-D semivariogram field in Fig. 4a along the line of zero vertical lag and $0-8 \mathrm{~m}$ horizontal lag (a horizontal slice); both exponential and hole-effect trends are isolated. Similarly, several distinct trends are evident in the migrated semivariogram field of Fig. 4b. The principal trends can be modeled with a transitional (exponential) structure
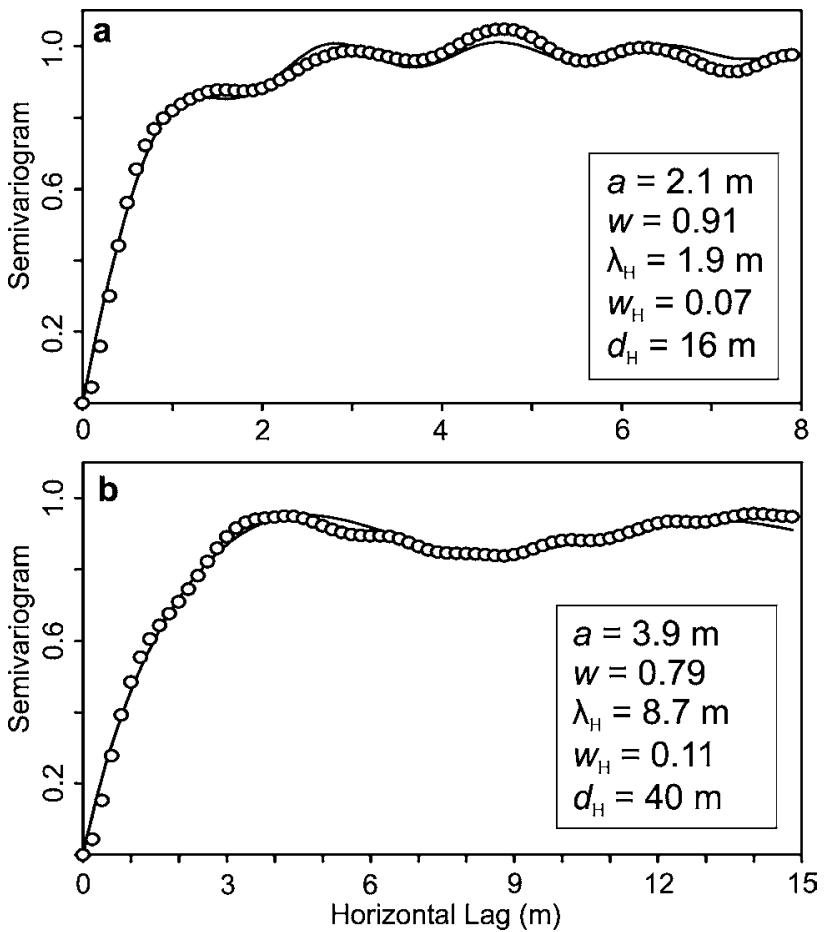

Figure 8. Horizontal semivariograms for migrated (a) $100 \mathrm{MHz}$ and (b) $50 \mathrm{MHz}$ reflection amplitudes. Points indicate experimental geostatistics for the depthconverted data; solid lines indicate least-squares models corresponding to the listed model parameters.

inclined a few degrees from the horizontal with a complementary periodic structure a few degrees off the vertical. These trends are representative of the near-horizontal reflections and the periodic nature of the GPR wavelet respectively. A secondary pattern is evident as the opposing ' $\mathrm{V}$ ' formations. The ' $V$ 's are not located at the origin due to strong interference by the principal transitional structure. However, less obvious exponential trends, parallel to the lobes of the ' $\mathrm{V}$ ', emanate from the origin with orientations of approximately $10-20^{\circ}$ from the horizontal. At positive horizontal and negative vertical lags, the ' $\mathrm{V}$ ' features represent a combination of dipping reflectors and diffraction hyperbola tails; at positive horizontal and positive vertical lags, they mainly represent diffraction hyperbola tails and thus, exhibit less persistence. When the depth-converted and migrated semivariogram fields are compared, a diminished diffraction contribution is evident as reduced strength of the ' $\mathrm{V}$ ' features, shrinking of the diffraction lobe, and reduced spatial persistence of the reflection/diffraction lobe as quantified by the exponential range parallel to the lobe (Fig. 4b). The reflection portion of the ' $V$ ' remains intact and steepens slightly consistent with migration.

The reflection data can be migrated at a variety of velocities and the semivariogram fields can be compared by 
Table 2. Frequency dependence of geostatistical parameters for the surface reflection data migrated at $0.095 \mathrm{~m} / \mathrm{ns} ; f_{\mathrm{a}}$ denotes antenna frequency; $f_{\mathrm{c}}$ denotes dominant received frequency; $\lambda_{\mathrm{c}}$ denotes approximate signal wavelength in the saturated zone; $\phi$ denotes principal correlation direction; the subscript 0 denotes the horizontal direction. Geostatistical model parameters are defined in the text; $n=0$ for all models.

\begin{tabular}{rccccccccccccc}
\hline \hline $\begin{array}{c}\boldsymbol{f}_{\mathbf{a}} \\
(\mathbf{M H z})\end{array}$ & $\begin{array}{c}\boldsymbol{f}_{\mathbf{c}} \\
(\mathbf{M H z})\end{array}$ & $\boldsymbol{\lambda}_{\mathbf{c}}(\mathbf{m})$ & $\boldsymbol{a}_{\mathbf{0}}(\mathbf{m})$ & $\boldsymbol{w}_{\mathbf{0}}$ & $\boldsymbol{\lambda}_{\mathbf{H} \mathbf{0}}(\mathbf{m})$ & $\boldsymbol{w}_{\mathbf{H} \mathbf{0}}$ & $\boldsymbol{d}_{\mathbf{H} \mathbf{0}}(\mathbf{m})$ & $\boldsymbol{\phi}\left({ }^{\circ}\right)$ & $\boldsymbol{a}_{\boldsymbol{\phi}}(\mathbf{m})$ & $\boldsymbol{w}_{\boldsymbol{\phi}}$ & $\boldsymbol{\lambda}_{\mathbf{H} \boldsymbol{\phi}}(\mathbf{m})$ & $\boldsymbol{w}_{\mathbf{H} \boldsymbol{\phi}}$ & $\boldsymbol{d}_{\mathbf{H} \boldsymbol{\phi}}(\mathbf{m})$ \\
\hline 200 & 85 & 1.1 & 1.2 & 0.95 & 1.8 & 0.02 & 48 & 2 & 1.2 & 0.95 & 2.1 & 0.02 & $\infty$ \\
100 & 68 & 1.3 & 2.1 & 0.91 & 1.9 & 0.07 & 16 & 3 & 2.2 & 0.91 & 2.1 & 0.07 & 15 \\
50 & 37 & 2.4 & 3.9 & 0.79 & 8.7 & 0.11 & 41 & 7 & 5.1 & 1.05 & NA & 0 & NA \\
\hline
\end{tabular}

slicing and modeling along common directions or along their respective principal angles. Results of this exercise illustrate the effects of migration and are summarized in Fig. 5 and Table 1. One of the main objectives of migration is the collapse of diffractions. Indeed, we see that any amount of migration results in an increase in modeled exponential range that is consistent with reduction of uncorrelated diffraction noise. This is true in both the horizontal and principal directions. In the vicinity of reasonable migration velocities, variation of the semivariogram parameters is within modeling uncertainty but the increase in exponential range is systematic and, as the data are over-migrated, the trend of increasing range becomes apparent even out to speed in air. This observation is perhaps counterintuitive as one might expect an image to move from uncorrelated, to correlated, to uncorrelated as the data are under-migrated, correctly migrated, and over-migrated respectively.

However, while the outcome of migration is dependent on many factors, such as the signal-to-noise ratio and the relative power in coherent reflectors versus scatterers, there are two inescapable results of migration that are consistent with the above observation. First, as migration velocity is increased, more energy is partitioned into the evanescent wave rather than the propagating wave (Claerbout, 1985), which results in less image energy, less image variability and increased correlation. Second, migration invariably results in a reduction of frequency content proportional to the square of the velocity (Yilmaz, 2001), which results in less image variability and increased correlation.

\section{Frequency Variation}

As apparent from Fig. 5, geostatistical model parameters are highly dependent upon GPR frequency. Migrated reflection data for antenna frequencies of 100 and $50 \mathrm{MHz}$ are shown in Fig. 6. Visually, as with all radar profiles, decreased antenna frequency results in lower resolution images that are less sensitive to small scale features such as thin bedding or scattering. Reduced resolution of the lower frequency reflection profiles is manifest as greater spatial ranges in the semivariogram fields shown in Fig. 7; horizontal slices and geostatistical models are shown in Fig. 8. Semivariogram fields for the lower frequency data are smoother and more continuous than those for the high frequency data and the ' $\mathrm{V}$ ' patterns are reduced in strength. In fact, for the $50 \mathrm{MHz}$ data, diffraction events are not recorded by the data (Fig. 6b) and accordingly, diffraction lobes are not evident in the $50 \mathrm{MHz}$ semivariogram field (Fig. 7b).

Quantitatively, the horizontal exponential range, horizontal hole wavelength, principal angle and principal exponential range all increase with a decrease in signal frequency as summarized in Table 2 and in Fig. 9. Specifically, the exponential range seems to scale almost linearly with the dominant received signal frequency for both migrated and non-migrated data (Fig. 9d).

A simple interpretation of the direct relationship between range and signal frequency is that the horizontal geostatistics are yielding information regarding the radar signal and not material properties. However, we are confident in the identification of reflection features such as dipping reflectors and diffractions in the semivariograms.

An alternative interpretation is that we are on some portion of the apparent correlation length versus measurement scale curve in the scale-triplet paradigm presented by Western and Blöschl (1999). The scale-triplet concept, illustrated in Fig. 10, suggests that finite measurement scales and data spacings independently lead to estimated correlation lengths greater than the true correlation length and that decreased data extent independently leads to estimated correlation lengths that are less than the true correlation length (where the correlation length is equal to one-third of the exponential range).

For radar reflection data, the measurement scale can be characterized by the Fresnel zone radius which defines the lateral radar reflection resolution (Annan and Davis, 1977). The Fresnel radius scales with the square root of the depth-wavelength product but is collapsed via migration to a theoretical minimum of one-half wavelength over the entire migrated domain (Yilmaz, 2001). Figure 11 illustrates the range-wavelength relationship. Since we observe a decrease in the estimated range with decreased signal wavelength (measurement scale), we can argue that the true range is no greater than the estimated range for the $200 \mathrm{MHz}$ data and 
Oldenborger et al.: Geostastistical Structure of Ground-Penetrating Radar Data

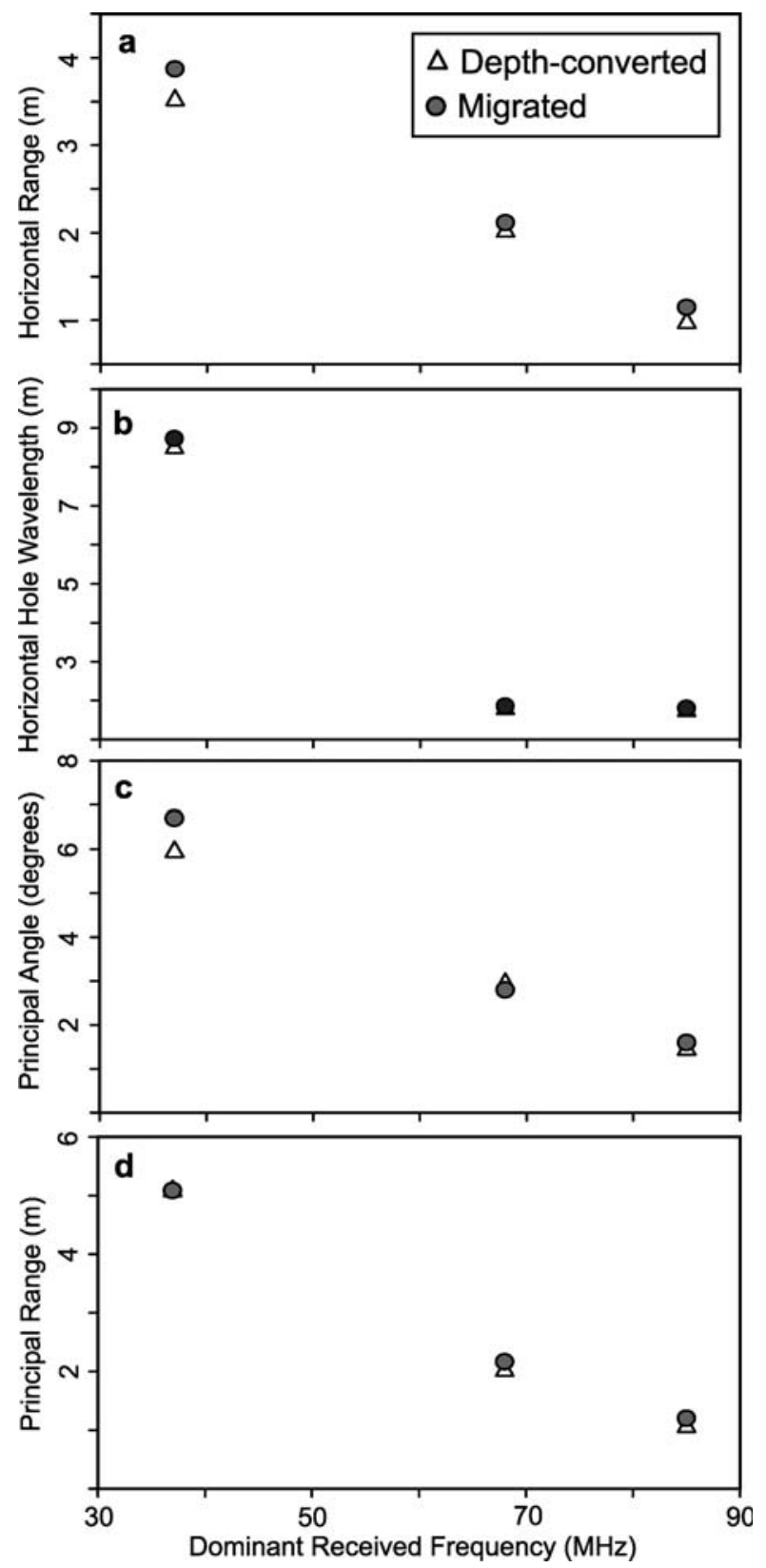

Figure 9. Effects of antenna frequency on (a) horizontal exponential range, (b) horizontal wavelength, (c) principal angle, and (d) principal exponential range for depth-converted (triangles) and migrated (circles) reflection amplitudes.

that the apparent decrease in range with decreased signal wavelength is a result of decreased measurement scale. However, in the unlikely event that the true range was on the order of the radar profile dimension, then our limited data extent would imply a gross underestimation of the true range according to Fig. 10. The effects of measurement scale and data extent can not be isolated without knowledge of the true range (Western and Blöschl, 1999).
Similar scaling behavior is observed for the variation of horizontal hole wavelength with frequency shown in Fig. 9b which exhibits threshold scaling behavior reminiscent of graphical representation of the representative elementary volume concept (Bear, 1972). The $50 \mathrm{MHz}$ hole wavelength is too large to be attributed to the periodicity of the individual dipping reflections and is thus an indicator of horizontal structural variation: information that is not obtained from the higher frequency data.

A final observation is that principal angle increases as frequency decreases (Fig. 9c) possibly due to an increase in relative strength and persistence of the dominant dipping reflector as frequency decreases. The increased strength of the dominant dipping reflector is observed by comparing the data shown in Figs. 3 and 6. Again, this is a result of a change in radar resolution.

\section{Discussion}

Due to the complex heterogenous nature of fluvial sand and gravel deposits, we observe directional complexity and interference patterns in the geostatistical analysis of GPR reflection data collected over such materials. Directional complexity results from the fact that significant reflection features are not necessarily horizontal or parallel and interference patterns arise in the case of multiple significant spatial correlation functions. Geostatistical structure will, of course, be site-specific; for the BHRS we are able to identify trends for near-horizontal reflectors, dipping reflectors and diffraction hyperbolas using the 2-D semivariogram fields.

However, it is not only the sedimentary materials and structures that dictate the geostatistics of the radar data; geostatistical trends of radar reflection amplitudes are a function of data processing, signal frequency. Raw or unprocessed reflection images may be strongly influenced by survey noise. Some data processing steps, such as gain and migration, increase image coherency and/or reduce noise in an attempt to produce a more accurate map of subsurface reflectivity. Other processing steps, such as dip filtering or horizontal low-pass filters, may also artificially increase or decrease image continuity. Such processing artifacts are manifest in the geostatistical structure of radar data and may be transferred to estimated hydraulic property fields.

The geostatistical structures of GPR data are also dependent upon the antenna frequency since the antenna frequency (along with material velocity) determines the radar signal wavelength which defines the measurement resolution. This behavior is well recognized in terms of qualitative GPR resolution, but, to our knowledge, has yet to be demonstrated geostatistically. Modeling the scale-dependence of GPR geostatistical parameters will require a complicated combination of both the scale-dependent properties of the natural 
Journal of Environmental and Engineering Geophysics
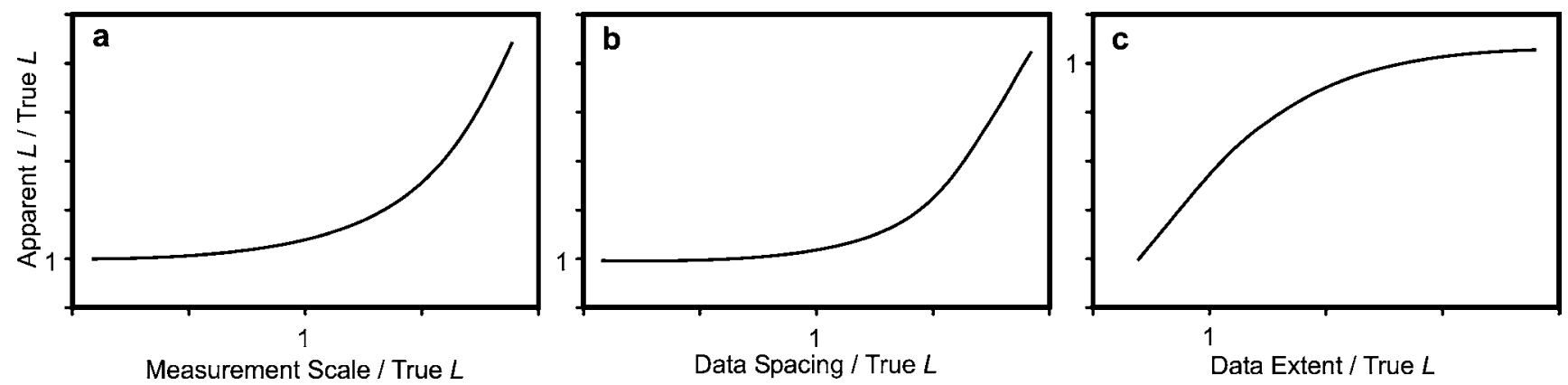

Figure 10. Independent effects of (a) measurement support, (b) data spacing and (c) data extent on the experimental correlation length $L$ (after Western and Blöschl, 1999). The correlation length is equal to one-third of the exponential range.

system and the frequency-dependent support volume of the measurement method (Beckie, 1996). Analysis using a scaletriplet paradigm (Western and Blöschl, 1999) helps isolate the scale-dependent effects of the signal and the material properties but requires appreciable knowledge about the spatial averaging functions and the true length scales of the variable in question.

Thus, the geostatistical structure of GPR data may be representative of the material or hydraulic properties, but only at a particular scale and only if processing artifacts can be neglected or accounted for. Variations with processing can be attributed solely to the modification of the data.

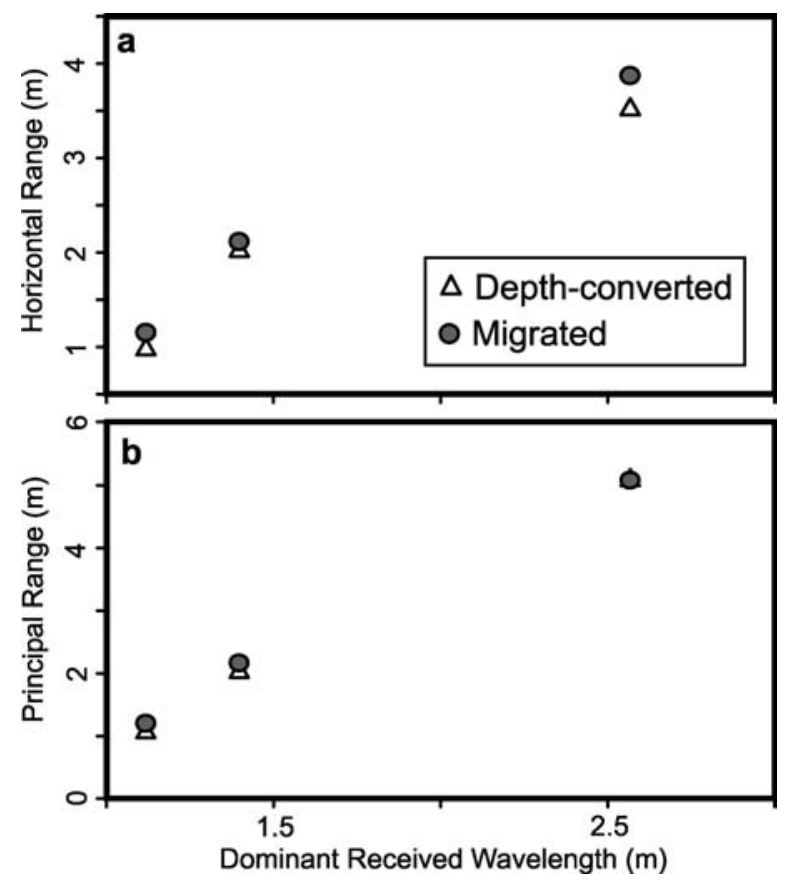

Figure 11. Effects of signal wavelength on (a) horizontal exponential range and (b) principal exponential range for depth-converted (triangles) and migrated (circles) reflection amplitudes.
However, variations with signal frequency involve signal properties, measurement support scale and the site-specific material properties.

Furthermore, in the event that effects of processing and frequency can be accounted for, the 2-D semivariogram fields contain an abundance of patterns that are not all accounted for since it is not always clear what every observed semivariogram pattern represents. That is, what physical subsurface features control specific reflections and how do those reflections show up in the geostatistics? While variations in processing help to identify and/or reduce the effects of some reflection features, synthetic reflection profiles might provide more insight into the translation of radar reflection features to the semivariogram field for a wider range of frequencies.

Given the variation of geostatistical structure with radar signal frequency and the uncertainty surrounding the information content of radar semivariograms, geostatistical treatment of radar reflection amplitudes seems unlikely to yield accurate estimates of material property distributions. Radar reflection surveys may be more appropriate for the estimation of sedimentary architecture (rather than material properties) possibly in a structural indicator fashion as presented by Langsholt et al. (1998), a cokriging scheme that employs reflector traveltime topography (Xu et al., 1992; Szerbiak et al., 2001), or in a more qualitative comparative database (Tercier et al., 2000).

\section{Conclusions}

Systematic geostatistical analysis of a single GPR reflection profile at several stages of data processing and three antenna frequencies reveals how spatial correlation structures depend on data processing and signal frequency.

Generally, the radar reflection data appear quite robust to the effects of reasonable data processing including gain functions and migration. For data collected at the BHRS, gain functions have the effect of removing a non-stationary trend from the non-gained data and a trace-specific gain 
Oldenborger et al.: Geostastistical Structure of Ground-Penetrating Radar Data

function increases semivariogram stability at large lags. Migration reduces the strength of diffraction features in the semivariogram fields and migration velocity exerts some control on the principal correlation angle. Principal exponential range increases only slightly after application of migration with a realistic velocity but over-migration results in a significant artificial increase of exponential range.

Signal frequency exhibits a more drastic influence on geostatistical structure. Geostatistical analyses of data collected with antennas of different frequency reveal consistent increases in both principal angle and spatial persistence as signal frequency decreases. Over the $50 \mathrm{MHz}, 100 \mathrm{MHZ}$ and $200 \mathrm{MHz}$ range of antennas, estimated exponential range is directly proportional to the dominant received wavelength which is directly proportional to the measurement resolution as quantified by the Fresnel zone limit of one-half wavelength.

The fundamental physical properties responsible for the observed geostatistics of radar reflection data and variation of those geostatistics with processing and frequency remain interesting research topics. However, regardless of physical explanations for observed geostatistical structures, the observed effects of processing and the difference between semivariogram fields for a single reflection profile at several frequencies have been demonstrated and must be considered before any application of surface reflection GPR in a geostatistical context.

\section{Acknowledgments}

Funding for this research was provided by the U.S. Army Research Office (grants DAAH04-96-1-0318 and DAAD19-00-10454.), the Natural Sciences and Engineering Research Council of Canada, the Inland Northwest Research Alliance, and the M.J. Murdock Charitable Trust. Access to the BHRS is made possible through cooperative agreements with the Idaho Transportation Department, the U.S. Bureau of Reclamation and Ada County. The authors would like to thank W.R. Peretti, W.P. Clement, L. Pearson and A. Collins for assistance with radar data acquisition, D. Lumley for discussions on migration, and two anonymous reviewers for helpful comments and criticisms.

\section{References}

Annan, A.P., 1999, Ground penetrating radar workshop notes: Sensors and Software Inc., Mississauga.

Annan, A.P., and Davis, J.L., 1977, Radar range analysis for geological materials: in Geol. Surv. Can. Report of Activities: 77-1B, 117-124.

Barrash, W., and Clemo, T., 2002, Hierarchical geostatistics and multifacies systems, Boise Hydrogeophysical Research Site, Boise, Idaho: Water Resour. Res., 38, No. 10, doi: 10.1029/ 2001WR001259.

Bear, J., 1972, Dynamics of fluids in porous media: Elsevier, New York.
Beckie, R., 1996, Measurement scale, network sampling scale, and groundwater model parameters: Water Resour. Res., 32, No. 1, 65-76.

Bracewell, R.N., 1986, The Fourier transform and its applications, 2nd ed.: McGraw Hill, New York.

Claerbout, J.F., 1985, Imaging the earth's interior: Blackwell Scientific, Oxford.

Deutsch, C.V., and Journel, A.G., 1998, GSLIB, geostatistical software library and user's guide, 2nd ed.: Oxford University Press, New York.

Fisher, E., McMechan, G.A., and Annan, A.P., 1992, Acquisition and processing of wide-aperture ground-penetrating radar: Geophysics, 57, No. 3, 495-504.

Gazdag, J., 1978, Wave equation migration with the phase-shift method: Geophysics, 43, No. 7, 1342-1351.

Gerlitz, K., Knoll, M.D., Cross, G.M., Luzitano, R.D., and Knight, R., 1993, Processing ground penetrating radar data to improve resolution of near-surface targets: in Proc. Symposium on the Application of Geophysics to Engineering and Environmental Problems, 561-574.

Greaves, R.J., Lesmes, D.P., Lee, J.M., and Toksöz, M.N., 1996, Velocity variations and water content estimated from multioffset, ground-penetrating radar: Geophysics, 61, No. 3, 683-695.

Isaaks, E.H., and Srivastava, R.M., 1989, An Introduction to applied geostatistics: Oxford University Press, New York.

Knight, R., 2001, Ground penetrating radar for environmental applications: Annu. Rev. Earth Planet. Sci., 29, 229-255.

Knight, R., Rea, J., and Tercier, P., 1996, Geostatistical analysis of ground penetrating radar data, a means of characterizing the correlation structure of sedimentary units: in EOS Trans. AGU, 77, No. 46, Fall Meet. Suppl., F237.

Langsholt, E., Kitterød, N., and Gottschalk, L., 1998, Development of three-dimensional hydrostratigraphical architecture of the unsaturated zone based on soft and hard data: Ground Water, 36, No. 1, 104-111.

Oldenborger, G.A., Schincariol, R.A., and Mansinha, L., 2003, Radar determination of the spatial structure of hydraulic conductivity: Ground Water, 41, No. 1, 24-32.

Olhoeft, G.R., 1994, Quantitative statistical description of subsurface heterogeneities with ground penetrating radar at Bemidji, Minnesota: U.S. Geol. Surv. Water Invest. Rep. 91-4034, 650-653.

Peretti, W.R., Knoll, M.D., Clement, W.P., and Barrash, W., 1999, 3-D GPR imaging of complex fluvial stratigraphy at the Boise Hydrogeophysical Research Site: in Proc. Symposium on the Application of Geophysics to Engineering and Environmental Problems, 555-564.

Rea, J., and Knight, R., 1998, Geostatistical analysis of groundpenetrating radar data, a means of describing spatial variation in the subsurface: Water Resour. Res., 34, No. 3, 329-339.

Szerbiak, R.B., McMechan, G.A., Corbeanu, R., Forster, C., and Snelgrove, S.H., 2001, 3-D characterization of a clastic reservoir analog, from 3-D GPR data to a 3-D fluid permeability model: Geophysics, 66, No. 4, 1026-1037.

Tercier, P., Knight, R., and Jol, H., 2000, A comparison of the correlation structure in GPR images of deltaic and 
barrier-spit depositional environments: Geophysics, 65, No. 4, 1142-1153.

Western, A.W., and Blöschl, G., 1999, On the spatial scaling of soil moisture: J. Hydrol., 217, No. 3-4, 203-224.

Woodbury, A.D., and Sudicky, E.A., 1991, The geostatistical characteristics of the Borden aquifer: Water Resour. Res., 27, No. 4, 533-546.
Xu, W., Tran, T., Srivastava, R.M., and Journel, A.G., 1992, Integrating seismic data in reservoir modeling, the collocated cokriging alternative: Soc. Petrol Eng. SPE 24742, $1-10$.

Yilmaz, Ö., 2001, Seismic data analysis, processing, inversion and interpretation of seismic data, investigations in geophysics No. 10: Soc. Exploration Geophysicists, Tulsa. 